\title{
Belphégor
}

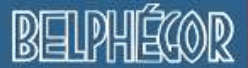

Littérature populaire et culture médiatique

13-1 | 2015

Distinctions That Matter/Fictions Économiques

\section{Mutation des supports, mutation des publics. La bande dessinée de la presse au livre}

Sylvain Lesage

\section{(2) OpenEdition}

Journals

Édition électronique

URL : http://journals.openedition.org/belphegor/628

DOI : 10.4000/belphegor.628

ISSN : 1499-7185

Éditeur

LPCM

Référence électronique

Sylvain Lesage, « Mutation des supports, mutation des publics. La bande dessinée de la presse au livre », Belphégor [En ligne], 13-1 | 2015, mis en ligne le 10 mai 2015, consulté le 01 mai 2019. URL : http://journals.openedition.org/belphegor/628; DOI : 10.4000/belphegor.628

Ce document a été généré automatiquement le 1 mai 2019.

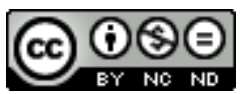

Belphégor est mis à disposition selon les termes de la Licence Creative Commons Attribution - Pas d'Utilisation Commerciale - Pas de Modification 4.0 International. 


\title{
Mutation des supports, mutation des publics. La bande dessinée de la presse au livre
}

\author{
Sylvain Lesage
}

1 En 1975, Luc Boltanski applique la théorie des champs tout juste élaborée par Pierre Bourdieu afin de décrire les mutations qui affectent le milieu de la bande dessinée ${ }^{1}$. Reprenant le concept bourdieusien, Boltanski analyse la structuration d'un champ de la bande dessinée sous l'influence conjuguée d'un certain nombre de facteurs, qui renvoient pour l'essentiel à l'apparition d'une nouvelle génération d'auteurs et de lecteurs. L'allongement des études, le bouleversement des structures socio-démographiques dans la France des Trente Glorieuses conduisent au déplacement des ambitions d'auteurs rejetant le modèle artisanal qui dominait jusque-là dans la bande dessinée, et affirment leur qualité d'auteurs à part entière.

2 Parallèlement, l'allongement des scolarités et un rapport décomplexé à la culture académique facilitent une mutation du lectorat français de la bande dessinée, marquée par la poursuite de la consommation de bande dessinée après l'enfance; Pilote serait le creuset et le paradigme d'une bande dessinée s'affranchissant de ses traits enfantins, pour s'adresser résolument à de grands adolescents, ou à de jeunes adultes. Au même moment, d'ailleurs, le succès médiatique colossal d'Astérix, véritable phénomène de société, pointe avec force ce déplacement des lectorats ${ }^{2}$. Enfin, le troisième pilier de cette recomposition du paysage de la bande dessinée se situerait dans la formation d'un appareil de consécration. L'apparition de générations de lecteurs familiers de la culture scolaire les pousse à reporter les outils d'analyse de ces formes savantes de la culture vers la bande dessinée et s'équipent rapidement en revues, conventions, salons, expositions, festivals, conférences...

3 Luc Boltanski proposait donc une lecture, à chaud, d'un processus aboutissant à une légitimation unique de la bande dessinée en France, lui conférant la dignité d'un «neuvième art » peu à peu reconnu par les instances de consécration de la culture officielle. 
4 L'analyse de Luc Boltanski a depuis été abondamment discutée et amendée ${ }^{3}$. Plus qu'une analyse entièrement convaincante des mutations qui affectent la bande dessinée, on peut y voir avant tout l'un des éléments qui construisent une polarité dans le paysage de la bande dessinée, en participant à l'affirmation d'une avant-garde résolument distincte d'une bande dessinée « commerciale ». L'article des Actes de la recherche en sciences sociales renforce presqu'autant qu'il analyse le mouvement de légitimation de la bande dessinée en France. Au moment même où il rédige son article, la France (et la Wallonie voisine) est pourtant affectée depuis plusieurs années par un processus profond de translation de la publication de bande dessinée depuis les pages des journaux vers celles des livres. Comment croire que la sacralité symbolique attachée au livre n'ait pas joué en faveur d'une dignité nouvelle pour la bande dessinée, particulièrement dans une vieille nation littéraire comme la France ? C'est ce rôle que nous voudrions évoquer ici, en fournissant quelques clés d'analyse d'un questionnement encore en chantier ${ }^{4}$.

\section{De la presse au livre : le basculement éditorial de la bande dessinée}

5 Le basculement du centre de gravité de l'édition de bande dessinée de la presse vers le livre est méconnu pour plusieurs séries de facteurs. La recherche spécialisée sur la bande dessinée, telle qu'elle est apparue dans le sillage de la structuration d'une communauté de fans (le fandom, ou domaine des fans), s'est évertuée à œuvrer pour la reconnaissance de la bande dessinée. À ce titre, le rôle des éditeurs s'est trouvé systématiquement négligé dans la mesure où il importait avant tout d'affirmer la valeur intrinsèquement artistique de la bande dessinée - l'art étant pensé, conformément à la doxa romantique, comme antinomique de considérations matérielles ou commerciales.

Ce basculement de la presse vers le livre a également été difficilement pris en compte parce que la première génération d'avant-garde décrite par Boltanski naît pour l'essentiel dans les pages de la presse. De Pilote à Métal Hurlant en passant par Ah! Nana, B.D. ou Fluide glacial, l'histoire des avant-gardes en bande dessinée constitue d'abord une succession de titres de presse, à lire les histoires de la bande dessinée issues de la démarche militante des fans. La construction d'une généalogie de la bande dessinée a en effet constitué l'une des dimensions centrales de l'entreprise de légitimation. Le dynamisme, l'inventivité, sont d'abord perçus dans le Journal de Mickey (1934), puis dans le Journal de Spirou d'aprèsguerre, celui d'un « âge d'or » qui verrait le sommaire structuré autour de Jijé, Franquin et d'autres; la création de Pilote marquerait, dans cette optique, un tournant, l'indice d'une moindre inventivité des journaux belges par rapport à leurs confrères français. Du Journal de Mickey à Pilote puis Métal hurlant ou (À Suivre), l'identification de jalons dans l'histoire française de bande dessinée passe d'abord par la mise en valeur de titres de la presse magazine.

7 Tout au plus se contente-t-on d'évoquer, dans ces panoramas généraux de la bande dessinée, un effondrement de la presse au tournant des années 1970-1980, voire au milieu des années 1980. Celui-ci est mal connu; pourtant, les chiffres de tirage des journaux spécialisés témoignent de l'ampleur du phénomène. Selon les données officielles de l'Office de justification de la diffusion, l'édition française de Spirou passe de 174000 exemplaires entre 1963 et 1966 à moins de 150000 exemplaires en 1969, et moins de 100000 en 1980. Sur la même période, Tintin passe de presque 260000 exemplaires en 
1963 à moins de 190000 en 1969, et s'est effondré à moins de 70000 exemplaires en 1982. Tous les journaux de bande dessinée ne sont pas également affectés par le phénomène ; significativement, les deux grands titres résistant bien au début des années 1980, PifGadget et surtout le Journal de Mickey (plus de 400000 exemplaires chacun en 1980) sont ceux qui se sont très peu adossés à un catalogue d'albums. Si ce choix de se cantonner à l'univers de la presse ne relève manifestement pas d'une stratégie délibérée, mais plutôt d'une succession d'erreurs éditoriales ne permettant pas d'investir le créneau rémunérateur de l'album, il n'en reste pas moins que cette politique permet sans doute de prolonger la survie des titres de presse, en particulier celle du Journal de Mickey.

En effet, l'effondrement des ventes de Spirou ne correspond nullement à l'effritement du lectorat des publications Dupuis. Dans le même temps, les ventes d'albums explosent: dans l'ouvrage hagiographique qu'il consacre au Journal de Spirou et aux éditions Dupuis, Thierry Martens, ancien rédacteur en chef et mémoire de l'entreprise, annonce une augmentation spectaculaire des ventes d'albums: «de trois millions d'exemplaires en 1970 à huit millions en $1980^{5}$ ». Les chiffres dont nous disposons concernant cette période, tirés des archives de l'entreprise, laissent apparaître une évolution moins spectaculaire sur le marché français. Mais le fait demeure: on assiste sans doute moins à un affaiblissement des titres de presse qu'à un changement de mode de lecture, qui se traduit par le déplacement du lectorat depuis le périodique vers l'achat d'albums.

Dans les années 1970, quelques titres de presse sont encore lancés. Éditeurs comme auteurs, accoutumés au support de presse, habitués à y voir l'horizon premier de la publication (sinon en termes hiérarchiques, du moins en termes chronologiques), continuent à se lancer dans la création de nouveaux titres. Mais à la fin des années 1980, l'échec de Métal hurlant (1987), de Corto et de Vécu (1989), l'agonie d'(À Suivre) et des différentes formules de Tintin (Tintin reporter, Hello Bédé) marquent la victoire définitive du livre sur la presse. La publication dans la presse ne présente plus dans la nouvelle économie éditoriale de la bande dessinée qu'un intérêt limité : l'abaissement du prix de vente, conjugué à l'extension du spectre des séries publiées en albums, fait de la presse un produit intermédiaire dont la rentabilité est de plus en plus incertaine.

Dans le même temps, la production d'albums explose ; en 1974, les éditeurs adhérant au Syndicat national des éditeurs déclarent publier 360 titres de bande dessinée ${ }^{6}$; dix ans plus tard, c'est plus de 1000 titres qui sont publiés annuellement par les membres du SNE. Le chiffre d'affaires généré par la branche édition d'albums accompagne cette progression spectaculaire : rapportée en euros de 2014, la production d'albums passe de 37 à 91 millions d'euros sur la même période.

11 Secteur éditorial de plus en plus rentable, la bande dessinée attire de plus en plus d'éditeurs. Au seuil des années 1950, seuls quelques éditeurs publient la bande dessinée sous forme d'albums, aux formes inspirées de l'album jeunesse. Il s'agit encore pour la plupart de publications isolées, où l'on peine à voir de véritables politiques éditoriales : ces publications correspondent avant tout à une démarche de rentabilisation d'un produit déjà amorti dans la presse. Les séries publiées ne sont, dans un premier temps, que celles plébiscitées par les lecteurs, et les éditeurs ne prennent alors pas un grand risque à sortir ces compilations. Alors qu'Hachette se retire du secteur malgré ses fortes positions des années trente, toute une génération d'éditeurs solidement implantés dans l'entre-deuxguerres se voit fragilisée par une transmission générationnelle délicate et le nouveau contexte suscité par l'adoption de la loi du 16 juillet 1949 sur les publications destinées à la jeunesse: Rouff, Tallandier, Garnier, Armand Colin ainsi que, dans une moindre 
mesure, Gautier-Languereau voire la Société parisienne d'édition accusent ainsi le coup, laissant la voie ouverte aux éditeurs belges. Dans les années 1980, ce paysage n'a plus rien de comparable: si une poignée d'éditeurs traverse la période (Dupuis, le Lombard, Dargaud, Casterman), les différences l'emportent, avec l'apparition d'une riche frange de petits éditeurs, souvent provinciaux, tentant de percer dans la bande dessinée, mais aussi de libraires-éditeurs, d'auteurs-éditeurs, et surtout l'arrivée de mastodondes des industries de la communication qui s'engouffrent dans ce segment du marché du livre, attirés par sa rentabilité.

Hachette, par exemple, après une longue éclipse de sa politique éditoriale en matière de bande dessinée, revient sur le devant de la scène à partir des années 1970, avant de se porter acquéreur de Dupuis en 1985. D’Albin Michel aux Presses de la Cité en passant par Gallimard ou Larousse, l'ensemble des maisons d'éditions françaises se prend d'intérêt pour la bande dessinée entre le milieu des années 1970 et le milieu des années 1980. L'arrivée sur le secteur de France-Loisirs ou de J'ai Lu et Pocket sonnent comme le signe d'une normalisation définitive de la bande dessinée dans l'industrie du livre.

\section{La bande dessinée et le livre : un transfert de sacralité}

La période des années 1950 aux années 1990 marque donc une transformation en profondeur de l'édition d'albums de bande dessinée, qui quitte son statut d'activité périphérique pour s'inscrire au cœur des recompositions de l'industrie éditoriale. Ce déplacement du centre de gravité de l'édition de bande dessinée depuis la sphère de la presse vers le monde du livre ne redistribue pas seulement les équilibres au sein du système éditorial. Bousculant les frontières, il fait entrer la bande dessinée dans une autre phase de son histoire. Renouant massivement avec le livre, elle retrouve un peu du prestige attaché aux albums de Töpffer, Cham ou des livres luxueux de Christophe. Plus largement, elle emprunte un peu de cette sacralité attachée au livre. En effet, comme l'a remarqué par exemple Emmanuèle Payen,

La longue histoire du livre a montré comment, particulièrement dans nos civilisations occidentales, l'apparition de la forme codique qui subsiste encore aujourd'hui a coïncidé avec les balbutiements de l'ère chrétienne, à tel point que l'objet s'est confondu pendant de nombreux siècles avec la parole divine qu'il avait devoir de transmettre, et en a revêtu les attributs. Lieu de la parole absolue, de la vérité et du pouvoir, le livre s'est d'abord développé dans une quasi-hégémonie comme forme tangible de l'existence de l'immatériel, l'espace d'une Révélation sans cesse proclamée - et transmise par le texte - puis comme l'étape incontournable dans la validation et la transmission des connaissances d'un univers qui reste à ordonner. Et quand, à la Renaissance, le lien entre la religion et le livre se distend, lorsque les écrits ne sont plus seulement religieux mais deviennent également traités de sciences, de philosophie, de médecine ou d'astronomie, le livre bénéficie encore d'assez d'aura pour rester l'instrument de légitimation du discours?'.

Le retour au livre contribue à donner à la bande dessinée une part de sa noblesse actuelle. Ce processus n'est pas sans conséquence sur le statut de la bande dessinée et sur la composition de son lectorat. Si l'adoption de la forme-livre donne un très net coup d'accélérateur au phénomène de légitimation du neuvième art, ce processus n'affecte cependant pas l'ensemble des formes de la bande dessinée, ni l'ensemble de ses publics.

Le passage de la bande dessinée dans la sphère du livre a indéniablement accru la légitimité que tâchaient alors de conquérir les militants du mouvement bédéphile. Les 
festivals, et en premier lieu celui d'Angoulême, ont joué un rôle majeur dans la structuration de l'action bédéphile, dans la reconnaissance du "neuvième art». Reprenant des rituels élaborés ailleurs, écartelé entre la tentation de Cannes (paradigme du rôle central d'un festival dans la légitimation d'un art) et celle du populaire, Angoulême a fortement contribué à forger une évidence au statut artistique de la bande dessinée. Angoulême participe de ces célébrations qui mettent en scène une dignité artistique en construction ${ }^{8}$.

Or ce qui est d'abord récompensé à Angoulême, ce sont des livres. Il est vrai que le prix le plus prestigieux, non prévu lors de la première édition, vient couronner un auteur, une carrière. Ce Grand Prix de la Ville d'Angoulême ne doit pas être pour autant l'arbre qui cache la forêt: des Alfred aux Fauves en passant par les Alph'arts, le festival s'est en permanence attaché à distinguer des livres. Les journaux, eux, occupent une place bien plus secondaire dans les palmarès. Car il ne faut pas oublier que le festival d'Angoulême s'intitule pendant très longtemps officiellement «salon »: le modèle est clairement le salon romantique. On met donc en avant les deux entités consolidées par les romantiques que sont l'auteur et son œuvre. Angoulême se trouve donc, dès les premières éditions, porté par un système de valeurs centré sur le livre. Les bédéphiles qui lancent et animent le festival y voient un moment pour se retrouver et échanger, mais aussi une occasion de fabriquer de la légitimité. Le discours sur les œuvres s'est alors greffé sur la valorisation des livres, pour contribuer à construire la figure de l'auteur de bande dessinée comme un artiste ${ }^{9}$.

17 Cette conquête de la légitimité culturelle passe également par les bibliothèques et les écoles. Ces instances de reproduction des hiérarchies culturelles ont, depuis le début du $\mathrm{xx}^{\mathrm{e}}$ siècle, nourri des critiques très vives à l'endroit de la bande dessinée, accusée de démoraliser la jeunesse et de la détourner de la lecture. Quand, dans les années 1960, le neuvième art entre progressivement dans les rayons des bibliothèques et dans les salles de classe, le support livre joue un rôle déterminant, qui permet de déjouer le vieux soupçon qui pèse sur les récits en images.

En France, les bibliothèques n'accordent que très tardivement une place aux productions destinées à l'enfance. Et, parmi celles-ci, ce sont les livres qui vont entrer en premier. Le journal, pas plus que le disque ou le jeu, n'a droit de cité dans ce temple du livre que constituent les bibliothèques françaises jusqu'aux années 1970-1980. Face à la demande pressante des enfants, constatant le succès des récits en images, les bibliothécaires infléchissent peu à peu leurs positions sur le neuvième art, avant que l'arrivée d'une nouvelle génération d'anciens lecteurs parmi les bibliothécaires ne bouscule les lignes. En se coulant dans les pages du livre, l'album a ainsi permis à la bande dessinée de se tailler plus rapidement une place dans les bibliothèques, pour des raisons à la fois formelles (solidité, circuits d'approvisionnements...) et symboliques. Devenu livre, la bande dessinée perd de son pouvoir de subversion aux yeux des gardiens des hiérarchies culturelles.

\section{La bande dessinée, entre neuvième art et infra- culture : de l'album au petit format}

Cette mue culturelle à laquelle s'est livrée la bande dessinée n'a cependant pas affecté l'ensemble des supports ni l'ensemble des lectorats de la même manière. Elle s'est en effet 
traduite par un éclatement du champ de la bande dessinée, avec une distinction croissante entre différents formats de publication. En façade, la conversion au livre de la bande dessinée fait entrer celle-ci dans une ère nouvelle, où elle jouit d'une légitimité inédite dans le paysage culturel européen et mondial. Devenue neuvième art, la bande dessinée bénéficie ainsi d'une respectabilité certes discutée, peut-être inaccomplie, mais dans tous les cas inédite sur le paysage européen.

Pourtant, pour qui observe de près le paysage éditorial de la bande dessinée, la situation de la bande dessinée français semble moins tranchée. En effet, ce double processus de basculement dans la sphère du livre et de légitimation du neuvième art s'accompagne d'un troisième terme: la relégation de la bande dessinée de "petit format » dans un « enfer » infra-culturel. Envers de l'album, envers du neuvième art, la bande dessinée de petit format condense les marques d'illégitimité. Qualité de fabrication médiocre, origine étrangère, anonymat fréquent, stéréotypie des personnages et des schémas narratifs... Isolément, aucune de ces caractéristiques ne distingue radicalement les petits formats de la bande dessinée "noble ", celle qui a droit de cité dans la presse et dans les pages des livres. C'est plutôt l'accumulation des stigmates qui signe le discrédit dont pâtissent ces publications.

21 Ce discrédit croissant, au moment même où la bande dessinée conquiert ses lettres de noblesse, peut s'observer notamment dans la virulence de la censure à laquelle ces petits formats sont soumis. L'examen des procès-verbaux de la Commission de Surveillance et de Contrôle instituée par la loi du 16 juillet 1949 sur les publications destinées à la jeunesse le montre clairement. Créée en large partie pour lutter contre l'influence jugée néfaste de la bande dessinée, la commission atténue peu à peu à la fin des années 1960 ses attaques vis-à-vis d'une production bourgeoise jugée tolérable, tandis que les formes "populaires » suscitent une inquiétude croissante. C'est là une constante de la censure que de peser d'un poids particulier sur les lectures du peuple, ou ce qui est censé en tenir lieu. Le peuple, les femmes, les enfants : trois formes particulières d'une même minorité culturelle constamment suspecte aux yeux des moralistes, incapables de penser ces catégories comme aptes à la distanciation ${ }^{10}$.

Malgré quelques alertes ponctuelles, Dupuis, Dargaud ou Casterman réussissent à éviter l'hostilité des commissaires. Certes, la paix obtenue par ces éditeurs est payée au prix fort, par l'affadissement de leurs journaux et des albums qui en sont tirés. Si les cas de censure restent rares, cela montre avant tout l'efficacité des mécanismes d'auto-censure, dans certains cas particulièrement spectaculaires (revolvers gouachés dans Spirou et les héritiers, par exemple) mais la plupart du temps intégrés au travail même des dessinateurs, qui s'interdisent situations, objets ou personnages comme susceptibles de leur procurer des démêlés avec la Commission de surveillance et de contrôle.

Celle-ci se montre plus soupçonneuse et moins encline aux compromis avec des éditeurs considérés comme plus vulgaires. L'examen de ses archives montre ainsi une relation nettement plus tendue entre la Commission et des éditeurs de petits formats tels que Lug à Lyon, ou Artima à Tourcoing. Artima pèse d'un grand poids sur le marché de la bande dessinée des années 1950 à la fin des années 1970 ; en 1964, la maison tourquennoise publie pas moins de 28 titres (Biggles, Commando, Météor, Tex Bill, Toni Cyclone, Vigor...) tirant pour l'essentiel entre 65 et 75000 exemplaires par numéro. Impéria, pour sa part, avec vingt-deux titres (Battler Britton, Garry Pacifique, Kit Carson, Panache, X 13...) dépasse fréquemment les 100000 exemplaires par titre. La Commission de surveillance et de contrôle des publications destinées à la jeunesse consacre, du milieu des années 1950 au 
milieu des années 1960, une part importante de son énergie à tenter d'influencer le contenu rédactionnel des publications d'Imperia et, surtout, d'Artima, convoquant à de multiples reprises Émile Keirsbilk, gérant des éditions Artima, pour tenter de l'intimider, suscitant nombre de sous-commissions ad-hoc pour tâcher de mettre au pas cette production de petit format.

Mais c'est indéniablement Elvifrance qui paie le plus fort le prix de la suspicion de la Commission à l'égard des lectures considérées comme "populaires ». Cette maison d'édition fondée en 1970 par Georges Bielec importe massivement des bandes dessinées italiennes qu'elle publie en France en petits formats. Démarrant son activité en 1970, elle se spécialise dans le créneau des publications "pour adultes ». Malgré cette mention figurant explicitement en couverture, la Commission mène une véritable guerre pour étouffer la production d'Elvifrance, contraint au dépôt préalable et soumis à une fiscalité confiscatoire.

On peut trouver comme signe de l'ampleur du discrédit culturel dont pâtissent les publications Elvifrance cet article anonyme publié dans le Nouvel Observateur du 2 octobre 1970, et qui tente d'attirer l'attention de la Commission de surveillance et de contrôle sur des publications jugées scandaleuses, en particulier Outre-Tombe :

Pour adultes français.

80000 exemplaires, 200000 lecteurs - Tous nécrophiles?

«Aah! mes belles garces, vous me rendez fou ! » râle Anatole, le garçon-morgueur, œil de verre, jambe de bois, avant de bondir sur la croupe nue de Fanchon, qui chevauche déjà un cadavre «si froid... si dur ». Nous n'en sommes qu'à la page 19. Il en reste une centaine. C'est la treizième livraison d'Outre-Tombe, une bande dessinée mensuelle «pour adultes ».

«Plus c'est dégueulasse, plus il y a de la violence et du cul, plus ça marche», explique le scénariste du Coffret des macchabées. Alors, pour faire marcher, il met le paquet : séance de nettoyage de cadavre (féminin, bien sûr) devant une " assistance de détraqués »; cérémonie secrète de l'ordre de Macchabée, dont l'âme apparaît en crevant le ventre ballonné d'une morte et en enflammant le jet de "gaz méphitique »...

Tirée à 60000 exemplaires - sans parler de l'édition italienne - cette bande dessinée n'a pas suffi à combler la demande. Le prochain Outre-Tombe sera « un numéro tout à fait exceptionnel où la mort, le sexe, la drogue et l'horreur se mêlent dans une étrange atmosphère, entre le rêve et la réalité ». Tirage prévu : 80 000. Ça fera dans les 200000 lecteurs. Qui peuvent être ces « adultes » ? Quel effet cathartique, quelle purge attendent-ils de ces représentations nécrophiliques?

Par le «langage de ceux qui n'ont pas de culture», comme dit Francis Lacassin, historien du "neuvième art ", la bande dessinée oppose Eros et Thanatos. Ainsi offre-t-on aux analphabètes fonctionnels les moyens de refouler leur agressivité latente dans la mise en spectacle du sadomasochisme.

Pour deux francs.

L'idée d'une bande dessinée "adulte" est manifestement totalement étrangère au journaliste ; mais, surtout, sa dénonciation d'Elvifrance repose sur un préjugé social très fort, faisant de ses lecteurs de véritables "analphabètes » : au moment où le discrédit culturel pesant sur la bande dessinée commence à s'alléger, il se trouve renforcé dans le cas des publications considérées comme "populaires». Au nom de la protection de la jeunesse, Elvifrance subit donc une censure d'une grande violence... sans que les dignitaires de la bédéphilie naissante, ou les auteurs qui s'imposent sur la nouvelle scène de la dignité culturelle, ne daignent s'élever contre ces procédés. Seule exception ou presque, Delfeil de Ton se livre à des plaidoyers vigoureux contre la censure exercée par le ministre de l'Intérieur, Raymond Marcellin contre la production d'Elvifrance. 

librairie comme un véritable segment de marché. Passé les premiers titres publiés par Losfeld, qui doivent peut-être leur interdiction autant au parfum de soufre de l'éditeur qu'aux ouvrages eux-mêmes, les commissaires ne s'insurgent guère contre les albums de bande dessinée érotique - tout en continuant à condamner les petits formats. Dans l'esprit de la commission, la différence de support justifie cette différence de traitement : un livre de bande dessinée érotique ne risque guère de tomber entre les mains d'un enfant - tandis qu'une revue vendue quelques francs pourrait, en dépit ou à cause de la mention « réservé aux adultes », attirer les enfants. Pravda la survireuse ou Epoxy, pourtant publiés par le sulfureux Éric Losfeld, ne posent guère problème à la Commission, comme l'indique le procès-verbal de la séance du 16 octobre 1968 concernant Epoxy: «Retraçant les aventures mythologiques d'une jeune femme que ses pérégrinations plongent dans une suite de situations scabreuses, cet album semble devoir coûter fort cher, ce qui en réduit sensiblement la nocivité pour les adolescents. On peut dès lors le considérer comme tolérable $»^{11}$. À l'inverse, le faible coût des publications Elvifrance, leur disponibilité en kiosque, mais également la faible qualité matérielle du format (papier, impression...) concourent à reléguer les petits formats dans un enfer culturel ${ }^{12}$.

a donc bien, à partir des années 1960 et surtout 1970, deux catégories de bande dessinée érotique : l'érotisme branché (Forest, Pichard, Lob, plus Manara et d'autres), publié dans des journaux d'avant-garde ou des livres luxueux, paré du parfum soufré d'une contre-culture socialement acceptable, parce que cantonné à la jeunesse petitebourgeoise - et, de l'autre côté, une pornographie populaire, souvent anonyme, figurant dans des formats sans noblesse, et représentant un véritable péril social. Cette construction de l'ordre social par les formats n'a rien de nouveau. C'était déjà l'argument développé par le procureur de la Seine dans son réquisitoire contre Boris Vian en 1950 :

Qu'on réédite [...] les œuvres de Gide sur vélin avec des estampes gravées sur cuivre, ou le Gamiani de Musset, sur papier couché à 20000 francs l'exemplaire, le mal n'est pas grand, il n'est pas profond, le livre ne risque guère de troubler l'âme de quelques bibliophiles avertis qui achèteront ces ouvrages. Mais le roman noir, mais J'irai cracher sur vos tombes, mais Les morts ont tous la même peau tirent à 50000 exemplaires, ces ouvrages se vendent 165 francs, le prix du paquet de cigarettes américaines, ils s'étalent à toutes les devantures ${ }^{13}$.

Ancienne, cette condamnation réactive, dans le cas de la bande dessinée, une construction sociale de l'espace des légitimités par le support de publication. Alors que la conversion d'une frange de la bande dessinée à la publication de livres contribue à alléger le poids des stigmates culturels, ceux-ci se trouvent à l'inverse redoublés pour cette bande dessinée « populaire » qui gagne, elle aussi, un lectorat adulte.

\section{Diversifications formelles : de l'album au livre}

Dans les années 1980, tandis que la presse continue de s'effondrer, la bande dessinée se fond de plus en plus étroitement dans le cadre du livre. En proposant formats et paginations inédits, en faisant dialoguer le neuvième art avec des formes littéraires, éditeurs et auteurs permettent de mettre à distance l'album tel qu'il avait été standardisé dans les décennies précédentes. Les éditions Futuropolis, lancées en 1974, s'imposent comme le fer de lance de ce nouveau mouvement. Une attention soutenue envers le livre et surtout une réflexion sur l'adéquation entre forme et récit conduisent ainsi l'éditeur à s'éloigner des standards, pour proposer des livres de grands formats (collection

Belphégor, 13-1 | 2015 
«30x40»), de petits formats (collection «X»), ou des paginations importantes («Copyright »). Le catalogue est travaillé selon une logique de collection affirmée, qui ne repose pas seulement sur des similarités narratives ou génériques, comme les grands éditeurs savaient le faire, mais également sur des choix formels affirmés : prédilection pour le noir et blanc, intense travail sur les maquettes, choix de papiers de qualité... Sous la houlette du graphiste Étienne Robial, la maison d'édition qui, significativement, constitue d'abord le prolongement d'une librairie, va donc transformer en profondeur l'art de faire du livre de bande dessinée. Là où ses prédécesseurs ne jouaient que sur des nuances (32, 48 ou 62 pages, cartonnage ou brochage...), Futuropolis ouvre l'espace des possibles, et fait entrer la bande dessinée pleinement dans l'âge du livre, en multipliant formats singuliers et jaquettes et en refusant de s'inscrire dans les cadres génériques de la bande dessinée, à commencer par la structuration en séries et la récurrence des héros. Ce mouvement est largement repris, prolongé, amplifié dans les années 1980, où l'on assiste à une diversification croissante des formats de publication. La collection « Romans (À Suivre) » représente un cas exemplaire de conversion d'un éditeur traditionnel à cette nouvelle donne. Éditeur de Tintin, de Petzi et des 4 As, Casterman, vénérable maison tournaisienne, se lance dans la deuxième moitié des années 1970 dans la bande dessinée pour adultes, reprenant les codes qu'a popularisés Futuropolis : noir et blanc, paginations volumineuses, valorisation des auteurs au détriment de la série... Ainsi, dès la parution en 1975 de La Ballade de la mer salée, récit de 163 planches se jouant des codes de la bande dessinée d'aventure, Casterman prend le contrepied de sa production précédente. Et, dans ses «Romans (À Suivre)», structurés par chapitres, l'éditeur contribue à faire tomber un peu plus les frontières entre livre et album. Dix ans plus tard, les Humanoïdes associés reprendront cette revendication littéraire en baptisant l'une de leurs collections « Roman graphique », terme qui en 1988 n'est pas encore une catégorie marketing.

31 À la fin des années 1980, alors que les dernières revues agonisent, le marché comme la dignité de la bande dessinée se sont transportés vers le livre. Avec quelques années de décalage, ce processus touche également le marché américain de la bande dessinée, où le livre constitue longtemps une rareté. Entre 1978 et la parution du symbolique Pacte avec Dieu de Will Eisner, et 1992, où Art Spiegelman reçoit un prix Pulitzer pour son Maus, un basculement sensible de l'édition de bande dessinée s'opère aux États-Unis. Dans la décennie suivante, ce basculement fera la fortune du graphic novel qui désigne avec éloquence ces livres de bande dessinée que l'on cherche à toute force à distinguer des comics $^{14}$.

\section{Conclusion}

L'album joue ainsi le rôle de catalyseur d'un mauvais genre, en art jouant les règles du jeu de la culture légitime. Le livre permet de désamorcer la suspicion dont la bande dessinée a pu être l'objet. Facilitant son entrée dans les bibliothèques, les écoles, les musées, elle permet également son incorporation rapide au champ de l'action culturelle étatique. Le corollaire de cette image nouvelle que le livre procure à la bande dessinée est la relégation du petit format dans un enfer infra-culturel. Censeurs, éducateurs mais aussi, plus largement, bédéphiles et historiens du neuvième art frappent d'un même dédain un format de publication qui constitue, aujourd'hui encore, un continent largement méconnu de l'histoire de la bande dessinée. L'histoire française de la bande dessinée s'est donc très largement structurée autour de l'histoire de quelques titres de journaux 
spécialisés emblématiques, mais également autour d'auteurs ou de séries passés par le livre. Le livre patrimonialise cette histoire de la bande dessinée, et le succès actuel des intégrales proposant la réunion de plusieurs volumes en un seul livre atteste que cette mémoire de la bande dessinée est toujours menacée d'effacement. C'est donc par le livre, toujours, que cette bande dessinée semble être vouée à s'inscrire dans une histoire. Cette histoire repose donc sur le double effacement de la bande dessinée publiée dans la grande presse (on ignore à peu près tout, par exemple, des séries publiées dans France-Soir, dont la diffusion était pourtant bien plus colossale que celle de Spirou et Tintin réunis) et des petits formats, envers culturel de ce «neuvième art » promis à l'album et «enfer » des censeurs et moralistes. Les historiens de la bande dessinée ont donc construit une barrière assez étanche entre les deux supports de publication, pour des raisons de légitimité. Pourtant, on peut entrevoir l'esquisse d'un changement de perception de cette production. La réédition en 2010 par Delcourt de Sam Bot, titre emblématique de la maison Elvifrance, à l'humour décalé et à l'argot truculent, semble ouvrir un début de normalisation pour ce patrimoine négligé. De même, le lancement, la même année, de la collection « $\mathrm{BD} \mathrm{Cul}$ » par les Requins marteaux peut constituer un signe que les frontières de la légitimité bougent. Qu'une maison d'édition emblématique de l'avant-garde s'empare des codes d'Elvifrance et joue avec eux au second degré pourrait ainsi constituer une revanche posthume d'un esprit Sam Bot sur les éteignoirs de la Commission de surveillance et de contrôle des publications destinées à la jeunesse.

\section{NOTES}

1. Luc Boltanski, "La constitution du champ de la bande dessinée », Actes de la recherche en sciences sociales, $\mathrm{n}^{\circ} 1,1975$, pp. 37-59.

2. Sur la construction éditoriale de ce "phénomène » et sa dimension multimédiatique, nous renvoyons à notre article "Astérix, phénomène éditorial. Du succès de librairie à la modernisation du marché de la bande dessinée en France », dans Bertrand Richet (éd.), Le tour du monde d'Astérix. Paris, Presses de l'Université Sorbonne-Nouvelle, 2011, pp. 13-21.

3. Voir notamment Éric Maigret, "La reconnaissance en demi-teinte de la bande dessinée », Réseaux, vol. 12, n 67, 1994, pp. 113-140, ainsi que du même, "Bande dessinée et postlégitimité " dans Éric Maigret, Matteo Stefanelli (dir.), La Bande dessinée : une médiaculture. Paris, Armand Colin / INA, coll. « Médiacultures », 2012, pp. 130-148.

4. Cet article a été initialement écrit pendant l'été 2012 ; les pistes qui sont ici évoquées ont été approfondies et, pour partie, nuancées dans l'aboutissement du chantier de recherche dont cet article ne proposait qu'un état des lieux : notre thèse de doctorat en histoire, soutenue en juin 2014 sous le titre L'Effet codex: quand la bande dessinée gagne le livre. L'album de bande dessinée en France de 1950 à 1990. Nous renvoyons à la version développée pour plus de précisions.

5. Thierry Martens, Le Journal de Spirou, 1938-1988 : cinquante ans d'histoire(s). Marcinelle, Dupuis, 1988, p. 171. Les chiffres cités cumulent les ventes d'albums dans tous les pays.

6. En dépit de son intitulé, ce syndicat regroupe également des éditeurs belges, présents à travers leurs filiales françaises. 
7. Emmanuèle Payen, « La Bibliothèque et l'œuvre, entre savoir et création », dans E. Payen (dir.), Les Bibliothèques dans la chaîne du livre, Paris, Éd. du Cercle de la Librairie, 2004.

8. Pour plus de détails sur le rôle d'Angoulême dans le processus de légitimation de la bande dessinée, nous renvoyons à notre article "Angoulême : 'la ville qui vit en ses images'? Politisation de la culture et institutionnalisation du festival», dans Anaïs Fléchet, Pascale Goetschel, Patricia Hidiroglou, Sophie Jacotot, Caroline Moine et Julie Verlaine (dir.), Une histoire des festivals: $\mathrm{XX}^{e}-\mathrm{XXI}{ }^{e}$ siècle, Paris, Publications de la Sorbonne, 2013, pp. 251-264.

9. On trouvera un écho grinçant dans les gags de la série Pauvre Lampil, de Cauvin et Lambil, qui mettent en scène un alter ego du dessinateur aux prises avec un système qui valorise la notoriété.

10. Sur ce point, voir notamment Jean-Yves Mollier, La Lecture et ses publics à l'époque contemporaine. Essais d'histoire culturelle, Le nœud gordien, Paris, PUF, 2001et Anne-Marie Chartier et Jean Hébrard (dir.), Discours sur la lecture, Paris, Bpi-Fayard, 2000. Voir également Jean-Yves Mollier, La Mise au pas des écrivains: l'impossible mission de l'abbé Bethléem au XXe siècle. Paris, Fayard, 2014.

11. AN, 1990208 - 6: archives de la Commission de Surveillance et de contrôle des publications destinées à la jeunesse, séance $n^{\circ} 77$ du 16 octobre 1968.

12. Jean-Matthieu Méon, «Illégitimité culturelle et censure de la pornographie. Le contrôle des bandes dessinées et des romans "pornographiques" en France depuis 1970 », dans Obscénité, pornographie et censure. Les mises en scène de la sexualité et leur (dis)qualification (XIXe-XXe siècles), édité par Régine Beauthier, Jean-Matthieu Méon et Barbara Truffin. Bruxelles, Éditions de l'Université de Bruxelles, 2010.

13. Propos cités par Martine Poulain, «La censure », dans Pascal Fouché (dir.), L’Édition française depuis 1945, Paris, Éd. du Cercle de la Librairie, 1998, p. 555-598.

14. Voir notamment sur ce point l'article de Jean-Paul Gabilliet, « Du comic book au graphic novel : l'européanisation de la bande dessinée américaine ", Image \& Narrative, $\mathrm{n}^{\circ} 12$, août 2005, consultable à l'adresse http://www.imageandnarrative.be/inarchive/tulseluper/gabilliet.htm. 